\title{
A case of osteochondritis dissecans of the lateral femoral condyle and patellofemoral joint surface occurring in the same knee
}

\author{
Yuichiro Maruyama • Kazuo Kaneko • Tomonori Baba
}

Received: 28 February 2013 / Accepted: 10 October 2013 / Published online: 24 October 2013

(C) The Author(s) 2013. This article is published with open access at Springerlink.com

\section{Introduction}

More than $70 \%$ of osteochondritis dissecans (OCD) lesions in the knee joint occur on the posterolateral surface of the medial femoral condyle, designated as the classic area. Approximately 15-20\% of OCD lesions in the knee joint occur in the inferior-central part of the lateral femoral condyle, and $1 \%$ or less occur on the patellofemoral joint surface $[1,2]$. We encountered a patient who simultaneously developed OCD in the inferior-central part of the lateral femoral condyle and in the patellofemoral joint surface.

\section{Case report}

A 14-year-old male who plays soccer presented with intermittent swelling and locking of the left knee joint over approximately the previous year. He had continued his routine of 3-h daily soccer practice, including running and jumping. On 19 February 2010, the patient's left knee pain worsened after soccer practice, and continued to worsen with any sporting movement. Effusion of his left knee joint was noted in a nearby hospital, and arthrocentesis was performed to aspirate a clear yellow fluid. Various conservative therapies were subsequently provided without success, at which point the patient was referred to our hospital.

On physical examination, the patient exhibited tenderness of the lateral aspect of the left knee and limited range of motion (ROM) from $30^{\circ}$ to $60^{\circ}$. No abnormal ligamentous instability of the knee was found. The McMurray test was negative for friction sounds, but caused recurrence of knee

Y. Maruyama $(\bowtie) \cdot$ K. Kaneko $\cdot$ T. Baba

Juntendo University Urayasu Hospital, Urayasu, Chiba, Japan

e-mail: you.maru@nifty.com pain. No instability of the patella was found. The patient was a healthy male, $170 \mathrm{~cm}$ tall and weighing $62 \mathrm{~kg}$, and he did not show abnormal alignment of the lower limbs, systemic bone disease, or hormonal abnormalities. A plain anteroposterior radiograph demonstrated a lucent region at the distal end of the lateral femoral condyle, and a lateral radiograph showed an additional lesion on the lateral surface of the trochlea in the patellofemoral joint (Fig. 1). Three-dimensional computed tomography (3D-CT) revealed a depression in the lateral femoral condyle and in the lateral surface the trochlea (Fig. 2). A T2-weighted MRI revealed high signal curvilinear lines at the same sites, with an image of synovial fluid flowing within the subchondral bone. A discoid lateral meniscus was also found (Fig. 3). Based on these findings, we suspected OCD and performed arthroscopic surgery on 25 March 2010.

During surgery, a shallow fissure was found slightly behind the lateral femoral condyle, but without evident peeling or instability of the cartilage surface. A depression was noted in the lateral patellofemoral joint as well, but without a fissure.

An arthroscopic plastic partial lateral meniscectomy was performed. Next, retrograde transarticular drilling was performed at five sites around each depression in the lateral femoral condyle and the patellofemoral joint (Fig. 4). Finally, a lateral patellar retinacular release was performed.

\section{Postoperative course}

The left leg was put in a knee brace and remained nonweight bearing for 3 weeks. Partial weight bearing was allowed after 3 weeks, and complete weight bearing after 6 weeks. ROM was limited to $0-30^{\circ}$ for 3 weeks, and then $0-90^{\circ}$ for the next 3 weeks. Three months after the operation, he had full ROM in the left knee. Participation in all sports was allowed 
Fig. 1 Preoperative anteroposterior radiograph demonstrating a lucent region at the distal end of the lateral femoral condyle (a), and lateral radiograph showing an additional lesion on the lateral surface of the trochlea in the patellofemoral joint (b)
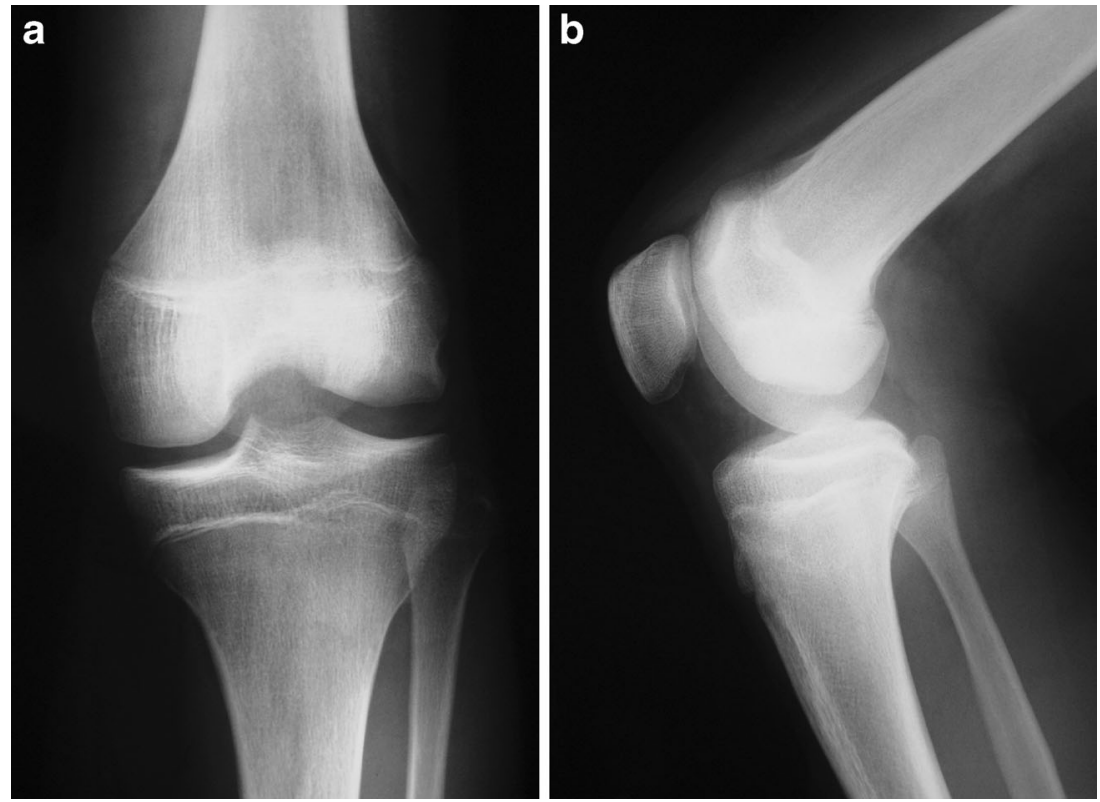

approximately 6 months postoperatively. The patient underwent rechecks with CT every 3 months after surgery. Although this led to frequent X-ray exposure, such a careful assessment was considered necessary because our understanding of postoperative management is insufficient in young patients compared with adults. We did not perform postoperative MRI because a number of studies have demonstrated the limited prognostic value of unenhanced MRI in OCD [3]. The depressions had disappeared on CT at 1 year postoperatively (Fig. 5). Currently, the patient has no pain or difficulty in his daily life or in sporting activities. On physical examination, his left knee ROM is $0-145^{\circ}$. There is no tenderness or appreciable effusion in the joint.

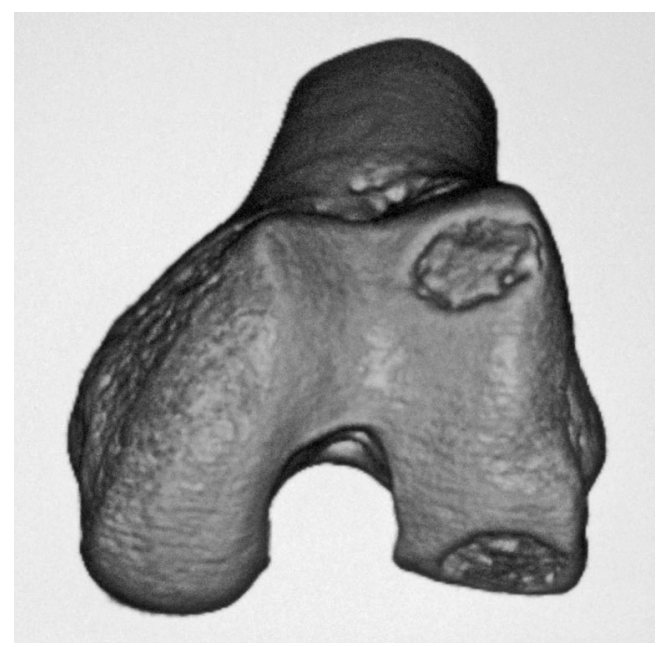

Fig. 2 Preoperative three-dimensional CT viewed from the front revealing a depression in the lateral femoral condyle and in the lateral surface of the trochlea

\section{Discussion}

Osteochondritis dissecans most commonly develops in the knee joint, accounting for $75 \%$ of cases, occurring most often in the lateral part of the medial femoral condyle. The etiology of OCD remains unknown, as the main causative factors (e.g., inflammation, ischemia, repetitive microtraumas, ossification disorders, and genetic defects) seem to vary among cases. There have been many reports in the literature regarding the potential role of trauma in the etiopathology of OCD. Repetitive microtrauma may induce stress that results in a stress fracture within the underlying subchondral bone. If repetitive loading persists and exceeds the ability of subchondral bone to heal, necrosis of the fragment may occur and lead to fragment dissection, separation, and nonunion [3]. Bilateral OCD is not rare, with a prevalence of $26 \%$ reported by Aichroth [1] and $18.5 \%$ reported by Smillie [4].

Reports of OCD occurring simultaneously at two sites in the same knee joint are limited. Examples include two cases with co-occurrence in the medial and lateral femoral condyles reported by Hanna et al. [5] and co-occurrence in the medial femoral condyle and patella in a single case reported by Kaya et al. [6]. Nathan et al. [7] described one patient who developed OCD three times in the same knee during a 4year period. In this case, OCD first occurred in the lateral femoral condyle, then in the medial femoral condyle, and finally in the trochlea. To our knowledge, there is no case report of OCD co-occurring in the lateral femoral condyle and the lateral surface of the trochlea patellofemoral joint surface.

It may be difficult to differentiate OCD from an ossification disorder in the distal femoral epiphysis. Caffey et al. [8] 
Fig. 3 Coronal (a) and sagittal (b) T2-weighted MRI slices revealing high signal curvilinear lines at the lateral femoral condyle and the lateral surface of the trochlea with an image of synovial fluid flowing within the subchondral bone
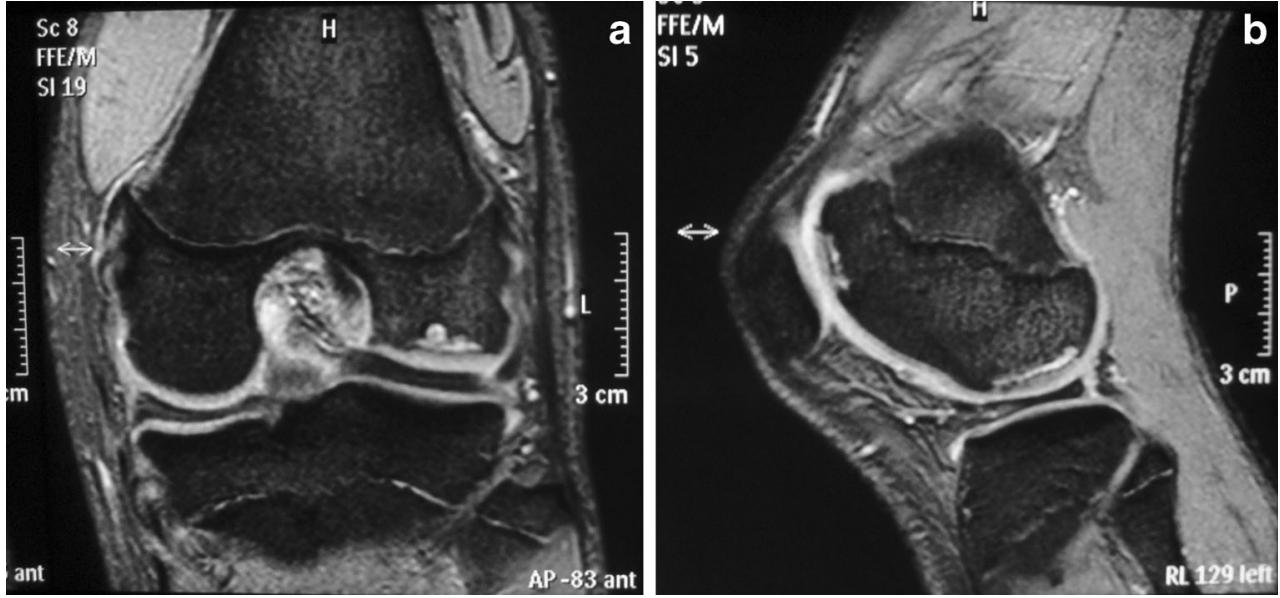

examined children between 3 and 13 years of age, and those aged 13 or older did not show radiographic signs of ossification disorders. As our patient was 14 years old at presentation, a diagnosis of OCD seemed plausible.

The lesions of OCD commonly heal spontaneously before epiphyseal closure. In this patient, knee pain had continued for a long period and MRI showed synovial fluid flowing into the subchondral bone, so spontaneous recovery was likely delayed. Drilling was performed to promote ossification and assimilation. Arthroscopic retrograde transarticular drilling of stable OCD lesions with a nearly intact articular surface has been well documented in the literature. On the other hand, antegrade transepiphyseal drilling without articular penetration may potentially injure the physis, leading to deformities. Antegrade drilling through the epiphysis avoids violating the articular surface and growth plate, but is associated with technical challenges of maintaining drill depth and placement accuracy.

Plastic meniscectomy was performed to reduce mechanical stimuli, and a lateral patellar retinacular release was carried out to relieve pressure against the patellofemoral joint. The depressions had almost completely disappeared on CT images by approximately 1 year postoperatively.

Because OCD of the lateral femoral condyle was associated with a discoid lateral meniscus in this patient, it is likely that frequent knee flexion and extension during sporting activities caused bending of the meniscus, resulting in increased stimuli to the cartilage of the lateral femoral joint surface, as reported previously [9]. In addition, this patient jumped frequently during soccer heading practice. We
Fig. 4 Arthroscopic findings: a shallow fissure was found slightly behind the lateral femoral condyle, indicated by the arrows (a); complete discoid lateral meniscus (b); after meniscoplasty (c); retrograde transarticular drilling was performed (d)
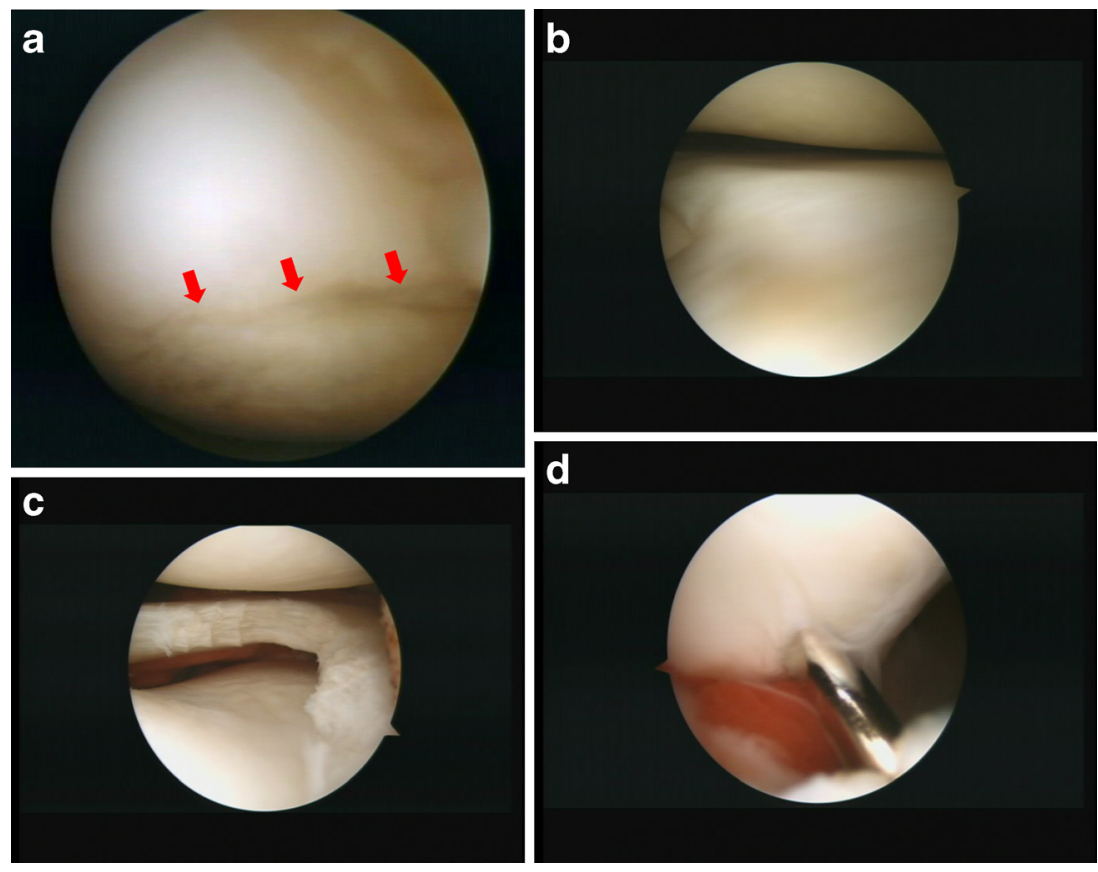


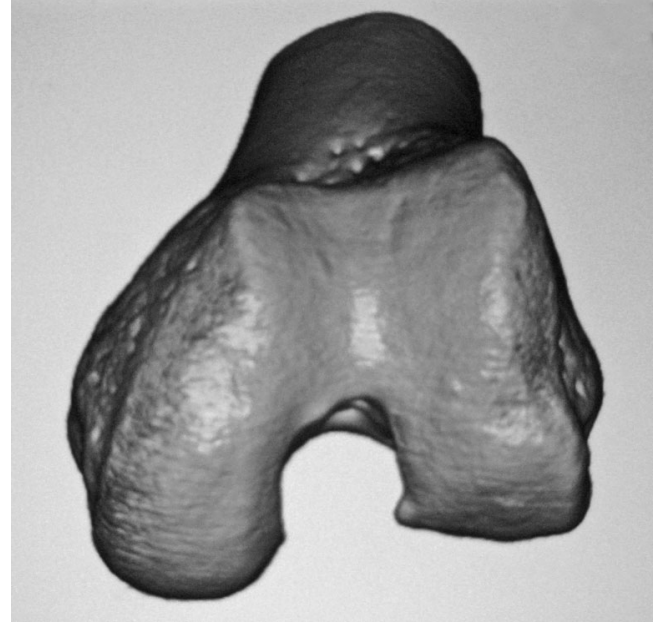

Fig. 5 The depressions had disappeared on 3D CT by 1 year postoperatively

concluded that repeated abrupt and strong knee extensions from a deep position had caused external deviation of the patella, adding strong pressure to the lateral femoral condyle, resulting in OCD of the patellofemoral joint surface [10-12]. Repetitive microtraumas are unlikely to be the sole cause of two lesions occurring in the same joint, and an underlying predisposition cannot be ruled out. However, the patient did not show abnormal joint instability, deformation of the lower limbs, or specific systemic diseases. Although hormonal changes during the growth period may slightly influence the process of cartilage growth and repair, the causative factors are poorly understood.

At present, 1.5 years postoperatively, the patient's clinical course is favorable (he can play sports normally without pain), but careful patient monitoring will be necessary, considering the potential for secondary occurrence of OCD not only in the knees, but also in other joints. Fortunately, his other joints, including the contralateral knee, were asymptomatic and we did not perform further radiologic evaluation.

Open Access This article is distributed under the terms of the Creative Commons Attribution License which permits any use, distribution, and reproduction in any medium, provided the original author(s) and the source are credited.

\section{References}

1. Aichroth P (1971) Osteochondritis dissecans of the knee. A clinical survey. J Bone Joint Surg 53-B:440-447

2. Schenck RC Jr, Goodnight JM (1996) Current concepts review. Osteochondritis dissecans. J Bone Joint Surg 78-A:439-456

3. Kocher MS, Tucker R, Ganley TJ, Flynn JM (2006) Management of osteochondritis dissecans of the knee. Current concepts review. Am J Sports Med 34:1181-1191

4. Smillie I (1957) Treatment of osteochondritis dissecans. J Bone Joint Surg 39-B:248-260

5. Hanna SA, Aston WJS, Gikas PD, Briggs TWR (2008) Bicondylar osteochondritis dissecans in the knee. A report of two cases. J Bone Joint Surg 40-B:232-235

6. Kaya A, Gucle B, Karaguven D, Benli IT, Ataogle O (2009) Osteochondritis dissecans occurring in the patella and medial femoral condyle in the same knee. Acta Orthop Traumatol Turc 43:72-76

7. Nathan L, Grimm BS, Breann Tisano BS, James L, Carey MD (2012) Three osteochondritis dissecans lesions in one knee. A case report. Clin Orthop Relat Res. doi:10.1007/s11999-012-2324-5

8. Caffey J, Madell SH, Royer C, Morales P (1958) Ossification of the distal femoral epiphysis. J Bone Joint Surg 40-A:647-714

9. Mitsuoka T, Shino K, Hamada M, Horibe S (1999) Osteochondritis dissecans of the lateral femoral condyle of the knee joint. Arthroscopy 15:20-26

10. Peters TA, McLean ID (2008) Osteochondritis dissecans of the patellofemoral joint. Am J Sports Med 28:63-67

11. Kurzweil PR, Zambetti GJ Jr, Hamilton WG (1988) Osteochondritis dissecans in the lateral patellofemoral groove. Am J Sports Med 16: 308-310

12. Takahashi Y, Nawata K, Hashiguchi H, Yamasaki D, Tanaka H (2008) Bilateral osteochondritis dissecans of the lateral trochlea of the femur: a case report. Arch Orthop Trauma Surg 128:469-472 